\title{
Pioneering small-group learning in Tanzanian emergency medicine: Investigating acceptability for physician learners
}

\author{
A G Lim, ${ }^{1}$ MD, MS; H Geduld, ${ }^{2}$ MB ChB, DipPEC, MMed, FCEM; K Checkett, ${ }^{3}$ MD; H R Sawe, ${ }^{4}$ MD, MBA, MMed; T A Reynolds, ${ }^{5}$ MD, MS, PhD \\ ${ }^{1}$ Division of Emergency Medicine, Department of Medicine, University of Washington, Seattle, WA, USA \\ ${ }^{2}$ Education and Training, Division of Emergency Medicine, Faculty of Health Sciences, University of Cape Town, South Africa \\ ${ }^{3}$ Section of Emergency Medicine, Department of Medicine, University of Chicago, Ill, USA \\ ${ }^{4}$ Emergency Medicine, Muhimbili National Hospital, Muhimbili University of Health and Allied Sciences, Dar es Salaam, Tanzania \\ ${ }^{5}$ Emergency and Trauma Care Programme, World Health Organization Department for Management of Noncommunicable Diseases, Disability, Violence and Injury Prevention, \\ Geneva, Switzerland; and Department of Emergency Medicine, University of California, San Francisco, CA, USA
}

Corresponding author: A G Lim (andrewglim@gmail.com)

Background. Emergency medicine (EM) is a relatively new, but growing medical specialty in sub-Saharan Africa. African EM training programmes have used small-group learning (SGL) modalities in their curricula. However, there is little knowledge of whether SGL modalities are perceived to be effective in these African EM training programmes.

Objectives. To investigate the acceptability of SGL for physicians' training in an academic Tanzanian emergency department using a novel EM curriculum.

Methods. Using responses to a written questionnaire, we explored the perceived effectiveness of SGL compared with traditional didactic lectures among 38 emergency department physician learners in Dar es Salaam, Tanzania. Perceptions of SGL were identified from qualitative responses, and regression analyses were used to determine strength of association between quantitative outcomes.

Results. Reported benefits of SGL included team building, simulation training, enhancement of procedural skills, and the opportunity to discuss opinions on clinical management. SGL scored more favourably with regard to improving clinical practice, enjoyment of learning, and building peer-to-peer relations. Lectures scored more favourably at improving medical knowledge. Preference towards SGL over lectures for overall training increased with years of clinical experience ( $95 \%$ confidence interval (CI) $0.16-0.62, p=0.002$, Spearman's rho 0.51 ), and the perception that SGL reinforces learner-teacher relationships correlated with seniority within residency training (95\% CI $0.14-0.86, p=0.007$, Spearman's rho 0.47 ).

Conclusion. Techniques of SGL were perceived as effective at improving clinical practice in the emergency department setting. These modalities may be more favourably accepted by more experienced physician learners - therefore, new EM teaching programmes in Africa should consider these factors when targeting educational strategies for their respective regions and learner cohorts.

\section{Context of emergency medicine training in sub-Saharan Africa}

Emergency medicine (EM) is a relatively new, but now established and growing medical specialty in sub-Saharan Africa. According to the African Federation of Emergency Medicine (AFEM), there are specialist-level graduates of EM training programmes from South Africa (SA), Ghana, Tanzania, and Ethiopia, with several new programmes with first generations of trainees in Rwanda and Botswana, among other African nations. ${ }^{[1]}$

Now that African EM has taken shape as a specialty-level discipline with academic and departmental leadership at major universities, new challenges have emerged for the continued success of the nascent field. A survey among recently graduated EM-trained specialists from the aforementioned African training programmes sought to characterise the challenges faced by the growing body of new EM practitioners. ${ }^{[2]}$ The largest perceived needs were the lack of leadership development and training, including materials for training and 'active learning', the need for improved relationships with faculty mentors, and the need for interprofessional communication training. Failure of the EM programmes to meet these needs has led to trainees leaving the field, a consequence noted by the first EM training programme developed in SA. ${ }^{[3]}$ A follow-up survey from the EM programme in Ghana highlighted the need for region-specific medical knowledge to enhance training curricula. ${ }^{[4]}$

Many of the EM curricula used in African training programmes were based on models from prior established residency programmes in North America, Europe, and Australia. These curricula consisted of multimodal approaches to learning, with the use of small-group learning (SGL), including case-based seminars, simulation training, and procedure-based skills labs to supplement traditional didactic lectures.

While there has been evidence supporting the effectiveness and acceptance of such modalities for their countries' respective learner populations, ${ }^{[5-11]}$ there has been little or no investigation regarding the appropriateness of these modalities in the sub-Saharan African context of EM training. SGL for EM has been favourably accepted by medical students in Botswana, ${ }^{[12]}$ but has not yet been explored in the postgraduate context. It cannot be assumed that western-developed educational modalities will be functionally (or culturally) appropriate for African educational norms, and investigations 
of learner perspectives would facilitate future programme design and curricular development for EM training on the continent.

In this study, we explore the acceptability and perceived effectiveness of SGL among physician-learners at the Muhimbili University Health and Allied Sciences (MUHAS) EM training programme in Dar es Salaam, Tanzania. These physician-learners include registrars (who have completed internship and have varying years of clinical experience, often transitioning to other specialty training or general practice by working for a limited time in the emergency department (ED)), EM residents (who have completed 1 - 3 years as registrars before joining the 3-year residency programme), and recent residency graduates $<1$ year out of training (now working as faculty at the ED at Muhimbili National Hospital (MNH)).

We compared the perceived effectiveness of SGL with lecture-based learning to investigate if acceptance correlates with learners' clinical experience or other demographic factors. Our objective was to identify characteristics of the Tanzanian physicians' experience with SGL modalities that could facilitate further development of EM residency curricula in other sub-Saharan African countries.

\section{Emergency medicine in Tanzania}

EM education was introduced in Tanzania in 2010 at MNH and has been challenged by the country's limited basic healthcare infrastructure and access to training, ${ }^{[13,14]}$ barriers exemplified in other sub-Saharan African countries. ${ }^{[15-17]}$ Regardless, the demand for improved prehospital and hospitalbased EM specialty training in this country continues to grow. ${ }^{[18]}$

Despite previous successes of SGL medical education in Tanzania, there have been few investigations of SGL acceptability in the region, and none for EM education. A recent study showed significant improvement to trauma resuscitation knowledge following implementation of a small-group-based resuscitation simulation course ${ }^{[19]}$ but researchers have yet to investigate the impact of SGL on other topics within the scope of EM postgraduate training.

The MUHAS EM curriculum, first introduced in 2010 with a primarily lecture-based format, was redesigned by faculty and residents in 2014 to formally incorporate SGL during educational conferences, comprising $\sim 40 \%$ of all conference time; this included case-based small-group seminars, procedure laboratories, and resuscitation simulations. The remaining $60 \%$ of conference time consisted of traditional lectures structured around monthly subject-based modules. The new 2014 curriculum provided the first exposure to non-lecture and non-bedside teaching modalities for many of its physician-learners. A core group of 6 - 10 specialist physician instructors (from Tanzanian, the US and SA medical schools) was primarily responsible for both lecture and SGL sessions, although visiting faculty from outside Tanzania gave occasional lectures supplemental to the core curriculum

Case-based small-group seminars (done on a weekly basis) involved groups of $4-6$ physician-learners with a senior resident or faculty facilitator, progressing through a patient presentation with discussions regarding clinical reasoning, diagnostic testing, therapeutic management and disposition decisions. Procedure laboratories (roughly once or twice per monthly module) included technical simulation of procedural skills, including diagnostic ultrasound scans, ultrasound-guided peripheral intravenous cannulation, advanced airway management and tracheal intubation. These were typically led by faculty and involved rotating groups of $4-6$ physician-learners. Resuscitation simulations were also done once or twice per monthly module and involved placing junior physician-learners as resuscitation leaders for management of emergent clinical scenarios (such as anaphylaxis, cardiac arrest, sepsis, trauma, and mass casualty triage) using low-fidelity mannequins, facilitator-controlled vital signs monitors, training cardioverter defibrillators, and other resources to simulate real-time, hands-on patient care.

\section{Methods}

We invited 38 physician-learners enrolled in the MNH EM training programme to participate in this study, to which all agreed. They represented all learners who had experienced the newly designed SGL curriculum at MNH's ED at the time of data collection in March 2014 (19 residents, 14 registrars, 5 recent residency graduates). A recent graduate, one of the authors of this study, was excluded. Informed consent was obtained for each participant at the time of interview and survey administration, and subjects were not required to participate in the study. Ethical approval was granted by respective review boards of MUHAS and the University of California, San Francisco (UCSF), USA.

We developed an exploratory survey using a written questionnaire to elicit both quantitative and qualitative responses through closed-and open-ended questions regarding the educational techniques used in the Muhimbili ED. Open-ended questions prompted subjects to characterise their perceptions of strengths and weaknesses of the various modalities of the SGL component of their education. To strengthen content and response process validity, the survey was designed and modified based on key informant interviews with Muhimbili ED faculty leadership and 10 of the participants. Faculty involved in AFEM curriculum development, and programme leaders of Tanzanian, SA and US EM residencies, also provided expert opinion to develop these instruments.

The 37-item written survey incorporated free response fields and Likertscale questions about physician-learners' perceptions of SGL within the MUHAS EM curriculum. The first section of the survey comprised openended questions about the effectiveness of SGL for both the acquisition of medical knowledge and improvement of clinical practice in the ED setting (Table 1). The second section asked respondents to directly compare SGL with lecture-based learning along several educational dimensions with numerical Likert-scale responses (Table 2). These educational dimensions were adapted from a study of Indian medical students' perceptions and acceptance of SGL v. lecture-based learning modalities, although this was originally intended for a general medical school curriculum not specific to EM education. ${ }^{[20]}$

To investigate whether the acceptance of SGL was associated with learner experience, we collected data on participants' years of clinical experience (number of years working in clinical settings following medical school), and their current level in the MUHAS EM training programme. Regression analyses were used to determine associations between learners' clinical experience and programme level with their acceptability of SGL based on the Likert-scale survey responses.

\section{Results}

The majority of the 38 respondents were male (63\%) and had $3-6$ years of clinical experience (81\%). Approximately half of respondents had some medically orientated SGL experiences prior to their EM education $(47 \%$ with experience, $45 \%$ without experience, $8 \%$ no response). Respondents 
were distributed among varying levels of advancement within the EM training programme (Table 1).

Responses to the qualitative section of the survey were categorised into major themes, providing insight into the overall acceptability of small-group education. The most commonly reported benefits of SGL included team building/relationship building in clinical settings $(n=13)$, the opportunity to discuss and interact with other learners $(n=10)$, the ability to simulate clinical scenarios $(n=9)$, the opportunity to clarify gaps in knowledge with faculty mentors $(n=7)$, improving clinical confidence $(n=6)$, and the enhancement of procedural skills $(n=6)$. The summary of these findings is reviewed in Table 2.

Table 3 summarises the Likert-scale responses regarding the perceived effectiveness of SGL compared with lectures. The majority of respondents agreed that SGL was both effective at improving medical knowledge (mean (standard deviation (SD) 4.18 (0.63), where a Likert response of $1=$ strongly disagree and $5=$ strongly agree $)$ and clinical practice $(4.14$ (0.85)). Overall, respondents felt that lectures were more effective at developing medical knowledge compared with SGL, but that the latter was comparatively more effective at improving clinical practice. SGL was preferred for enjoyment of learning and building positive peer-to-peer relations. For overall training, small groups and lectures were evenly favoured.

There was a significant and positive correlation between clinical experience and preference towards SGL for overall training compared with lectures (coeff $0.39,95 \%$ confidence interval (CI) $0.16-0.62, p=0.002$, $R^{2}=0.30$; Spearman's rho $0.51, p=0.003$ ). There was also a significantly positive correlation between level of EM residency training and the perception that SGL reinforces learner-to-teacher relationships (coeff. $0.48,95 \%$ CI $0.14-0.86, p=0.007, R^{2}=0.21$; Spearman's rho $0.47, p=0.005$ ).

\section{Discussion}

The findings of the study suggest that SGL methods implemented in a novel Tanzanian EM training curriculum had favourable learner acceptability for improving clinical practice, enhancing enjoyment of learning, and reinforcing peer relations, while lectures were favoured for improving medical knowledge.

The following themes emerged in our study with regard to the strengths and weaknesses of SGL compared with lectures:

- SGL was preferred over lectures for overall training by participants with more clinical experience. The association between years of clinical experience and preference for SGL was consistent with the literature, showing that physician-learners are more satisfied with learning environments than approximate clinical practice, especially when further removed from the medical school experience. ${ }^{[21]}$

- SGL reinforces learner-to-learner relationships compared with lectures. SGL appeared to reinforce teamwork and relations among learners, especially in clinically applied settings.

- SGL reinforces learner-to-teacher relationships for participants in continued residency training. As learners progressed through the residency programme, they identified SGL as more effective at building relations with their teachers. This could be owing to the trust built over time with mentors, along with the camaraderie fostered through residency. Additionally, experienced trainees were expected to participate as teachers/leaders in both SGL and lectures, and were therefore more likely to identify with
Table 1. Participants' gender, clinical experience and training level $(N=38)$

\begin{tabular}{ll}
\hline Demographics & $\boldsymbol{n}(\%)$ \\
\hline Gender & \\
$\quad$ Male & $24(63)$ \\
Female & $14(37)$ \\
Clinical experience, post-medical school (years) & \\
3 - 4 & $16(42)$ \\
5 - 6 & $15(39)$ \\
7 - 8 & $7(18)$ \\
Level of EM training & \\
Registrar & $14(37)$ \\
Resident, year 1 & $9(24)$ \\
Resident, year 2 & $4(11)$ \\
Resident, year 3 & $6(16)$ \\
New faculty (year 4) & $5(13)$
\end{tabular}

Table 2. Summary of participants' perceptions of SGL $(N=38)$

Effective aspects of SGL

Teambuilding and peer-relationship building $(n=13)$

Opportunities for discussion/interaction with other learners $(n=10)$

Opportunities for practising real-life clinical scenarios $(n=9)$

Opportunities to clarify gaps in knowledge with mentors $(n=7)$

Improves confidence of clinical skills $(n=6)$

Interactivity of seminar to learn physical/procedural skills $(n=6)$

Improvement of public speaking/communication/presentation skills ( $n=5)$

Improves critical thinking and medical concepts $(n=4)$

Ineffective aspects of SGL

Small groups can be too much like mini-lectures $(n=2)$

Small-group teaching should be divided by trainee level $(n=2)$

Table 3. Perceived effectiveness of lectures v. SGL $(N=38)$

\begin{tabular}{ll}
\hline Characteristic of learning modality & Mean (SD) \\
\hline Improving medical knowledge & $2.20(1.15)$ \\
Improving clinical practice & $4.12(0.86)$ \\
Ability to organise what you've learned & $3.79(0.96)$ \\
Defining/clarifying of learning objectives during lesson & $2.82(1.31)$ \\
Developing clinical/diagnostic reasoning & $3.24(1.35)$ \\
Developing independent critical thinking & $3.45(1.42)$ \\
Enjoyment of learning & $4.15(0.87)$ \\
Motivation to learn more on your own & $3.27(1.31)$ \\
Reinforcing learner-to-learner relationship building & $4.12(0.88)$ \\
Reinforcing learner-to-teacher relationship building & $3.53(1.35)$ \\
Overall training & $3.45(1.09)$ \\
${ }^{*} 1=$ lectures much more effective; $2=$ lectures more effective; $3=$ neutral; \\
$4=$ small groups more effective; $5=$ small groups much more effective.
\end{tabular}

mentorship roles. Conversely, registrars and 1st-year residents may have felt more distanced from the small-group experience and hesitant of teacher-learner interactions, perhaps owing to nascent professional relations with their mentors. Some younger participants expressed feeling intimidated to speak out in the early years of medical school, a feeling they may have carried over to their postgraduate training. 
There were also negative aspects to the SGL modalities that were expressed by the physician-learners. Interestingly, small groups were criticised when seminars were too 'lecture-like' in quality, i.e. when they failed to retain features unique to SGL. Further criticism of small groups occurred when learners were intermixed with those from other training levels. This resulted in decreased ability to form team bonds, given that the experience and knowledge among peers was discordant.

\section{Study limitations}

There were significant limitations to this study. It was a single-site study, affecting the generalisability of our findings to other African EM training programmes. Although we used a total sample of physician-learners with experience in EM-specific SGL in the only EM specialty training programme in Tanzania, the sample size was still small and thus limited in statistical significance. Our analyses would benefit further from longitudinal studies of this cohort as they progress through the curriculum.

An attempt was made to calculate effect size between the resident and registrar cohorts for their overall preference of SGL v. lectures (using Cohen's $d$ ). However, these calculations showed no significant effect size difference. Further, no significant associations were found with gender and other independent variables.

Despite attempts at ensuring the appropriateness of survey items via key informant interviews and pretesting, the instruments had not been previously validated or standardised, in part due to inability of finding existing instruments suitable for the research objectives. Therefore, specific attempts at validity testing for the quantitative portion (such as inter-item reliability) were not pursued. We solicited learner opinions of a better conduit for medical knowledge, and did not seek objective measures of medical knowledge; therefore, participants' perceptions were undoubtedly influenced by their previous learning experiences. Furthermore, some of the participants (senior residents) also had teaching responsibilities, e.g. to lecture or facilitate small groups; this bias could not be further controlled in our analysis.

The significance of SGL being associated with improvement of clinical practice and lectures with medical knowledge deserves further exploration. The qualitative data would suggest that clinical practice entails practical skills and the ability to perform patient care at the bedside. It is not entirely clear from the survey data if clinical practice also encompassed provider confidence, team building, communication, critical awareness, or other essential skills related to patient care. Likewise, medical knowledge may have meant basic science knowledge, clinical science knowledge, or other fundamentals of medical science; these findings require a more nuanced investigation in future research.

\section{Conclusion}

Physician-learners with more clinical experience and professional maturity tended to be more accepting of SGL as a learning modality. Residency programmes should consider these factors when deciding on which educational modalities to incorporate into their curricula. As EM educators in Africa tailor their residency programmes' educational curricula to their regional needs, they should weigh factors such as clinical experience of their trainees and potential benefits to team building and learning enjoyment when deciding how to incorporate SGL modalities.
SGL has been a prominent feature of North American, European, and Australian EM training in recent decades. With the calls toward 'novel' forms of education developed in these countries, we must be careful in assuming that these learning methods will be culturally or socially appropriate for a given region's needs in the African context. ${ }^{[22]}$ If we lack an understanding of the educational context where a new training curriculum is being implemented, the curriculum could easily fail to have its desired effect. We believe that medical practitioners who are not familiar with the relevant country's educational context must also have a strong understanding of regional educational issues, or must be working in collaboration with educational researchers well versed in the regional context.

For example, it is possible that perceptions and acceptability of SGL are different among EM residents in the USA, where the average postgraduate time period out of medical school is only $1-2$ years. Given that the majority of Tanzanian physician-learners were in their 5 th through 8 th postgraduate year, this differential in experience level alone provides evidence that African EM curricula need to be designed with its unique learner populations in mind. There are also considerable differences in the nature of clinical experiences between the average Tanzanian and US physician-learners entering EM training.

Nonetheless, this research does suggest that SGL may address some of the needs identified by Bae et al. ${ }^{[2]}$ in their survey of African EM specialists. SGL may be effective in increasing relationship building between learners and mentors, promoting active learning, and building more effective leadership and communication training. However, more targeted research, and programme-specific monitoring and evaluation of the curriculum, will be needed to explore these themes further for the future development and strengthening of EM education in sub-Saharan Africa.

Acknowledgements. We would like to acknowledge Prof. V Mwafongo, MUHAS, and head of EM at MNH. We thank all participants, physicians, nurses, and other ED staff who work tirelessly on behalf of their patients, and for the advancement of EM in Tanzania.

1. African Federation of Emergency Medicine. 2016: http://www.afem.org (accessed 21 December 2016). 2. Bae C, Geduld H, Wallis LA, Smit DV, Reynolds T. Professional needs of young emergency medicine specialists in Africa: Results of a South Africa, Ethiopia, Tanzania, and Ghana survey. Afr J Emerg Med 2016; 6(2):94-99. http://dx.doi.org/10.1016/j.afjem.2016.02.005

3. Wen LS, Geduld HI, Nagurney JT, Wallis LA. Africa’s first emergency medicine training program at the University of Cape Town/Stellenbosch University: History, progress, and lessons learned. Acad Emerg Med 2011; 18(8):868871. http://dx.doi.org/10.1111/j.1553-2712.2011.01131.x

4. Martel J, Oteng R, Mould-Millman NK, et al. The development of sustainable emergency care in Ghana: Physician, nursing and prehospital care training initiatives. J Emerg Med 2014;47(4):462-468. http://dx.doi.org/ 10.1016/j.jemermed.2014.04.041

5. Maddry JK, Varney SM, Sessions D, et al. A comparison of simulation-based education versus lecture-based instruction for toxicology training in emergency medicine residents. J Med Toxicol 2014;10(4):364-368. http:// dx.doi.org/10.1007/s13181-014-0401-8

6. Li CH, Kuan WS, Mahadevan M, Daniel-Underwood L, Chiu TF, Nguyen HB (ATLAS Investigators: Asia neTwork to reguLAte Sepsis care). A multinational randomised study comparing didactic lectures with case scenario in a severe sepsis medical simulation course. Emerg Med J 2012;29(7):559-564. http://dx.doi.org/10.1136/ emermed-2011-200068

. Wang EE, Beaumont J, Kharasch M, Vozenilek JA. Resident response to integration of simulation-based education into emergency medicine conference. Acad Emerg Med 2008;15(11):1207-1210. http://dx.doi.org/10.1111/j.1553 2712.2008.00208.x

8. Häske D, Beckers SK, Hofmann M, et al. The effect of paramedic training on pre-hospital trauma care (EPPTC study): A study protocol for a prospective semi-qualitative observational trial. BMC Med Educ 2014;14(1):32. http://dx.doi.org/10.1186/14/2-6920-14-32

. Chung SP, Cho J, Park YS, et al. Effects of script-based role play in cardiopulm

.

$\mathrm{L}$, Peck Breaking bad news education for emergency medcine residents: A novel training module using simulation with the SPIKES protocol. J Emerg Trauma Shock 2010;3(4).385-388. http://dx.doi.org/10.4103/0974-2700.70760

R, Langdorf MI, Lotfipour S. Prospective randomized crossover study of simulation vs. didactics for teaching medical students the assessment and management of critically ill patients. J Emerg Med 2011;40(4):448-455. http://dx.doi.org/10.1016/j.jemermed.2010.02.026

school - experiences from Botswana Afr J Emerg Med 2013:3(4):157-163. http//drdoi org/10.1016/jafem 2013.04 .003 
13. Reynolds TA, Mfinanga JA, Sawe HR, Runyon MS, Mwafongo V. Emergency care capacity in Africa: A clinical and educational initiative in Tanzania. J Public Health Policy 2012;33(Suppl 1):S126-S137. http://dx.doi. org/ $10.1057 /$ jphp. 2012.41

4. Hsia RY, Mbembati NA, Macfarlane S, Kruk ME. Access to emergency and surgical care in Sub-Saharan Africa: The infrastructure gap. Health Policy Plan 2012;27(3):234-244. http://dx.doi.org/10.1093/heapol

czr023 N, Chandra A, Kestler A. Development of emergenc
2011;1(3):108-112. http://dx.doi.org/10.1016/i.afjem.2011.08.002

2011,1(3).108-112. htp.//4x.doi.org/10.1016/j.afjem.2011.08.002 Henya. Afr J Emerg Med 2011;1(4):160165. http://dx.doi.org/10.1016/j.afjem.2011.10.008

7. 71.
18. Celletti F, Reynolds TA, Wright A, Stoertz A, Dayrit M. Educating a new generation of doctors to improve the health of populations in low- and middle-income countries. PLoS Med 2011;8(10):e1001108. http://dx.dol. org/10.1371/journal.pmed.1001108

. Bergman S, Deckelbaum D, Lett R, et al. Assessing the impact of the trauma team training program in Tanzania J Trauma 2008;65(4):879-883. http://dx.doi.org/10.1097/TA.0b013e318184a9fe

2. Nanda B, Manjunatha S. Indian medical students' perspectives on problem-based learning experiences in the undergraduate curriculum: One size does not fit all. J Educ E org/ $/ 10.3352 /$ jeehp. 2013.10 .11

1. Al-Azri H, Ratnapalan S. Problem-based learning in continuing medical education: Review of randomized

2. Bleakley A, Brice J, Bligh J. Thinking the post-colonial in medical education. Med Educ 2008;42(3):266-270. http://dxdoiorg/10.1111/j.1365-2923.2007.02991 